\title{
Antimicrobial pattern in neonatal intensive care unit (NICU) in a tertiary care hospital
}

\author{
Ajay $\mathrm{J}^{1}$, Abhishek Pate $\mathrm{l}^{2 *}$, Govind Auradkar ${ }^{3}$ \\ ${ }^{1,2}$ Assistant Professor, ${ }^{3}$ Senior Resident, Department of Pediatrics, Navodaya Medical College, Raichur, Karnataka, India. \\ Email: ajayj1987@gmail.com
}

Abstract Background: Antimicrobials are the most common medications used in NICU. Most often they are prescribed as a part of treatment for sepsis. They are also administered empirically in neonates during their hospital stay even without sepsis. The western countries have moved from empirical approach to evidence based approach but the literature review in developing countries shows the prevalence of irrational prescription of antibiotics because of empirical approach which is driven by the economic constraints of the patients. Objectives: To study the pattern of antimicrobial (AMA) drug use in neonates admitted to NICU. Methods: This is a retrospective observational study conducted in a tertiary care hospital in south India. The data was collected retrospectively and all the neonates treated with antibiotics were included in the study Results: Out of the 102 neonates admitted $76(74.5 \%)$ received antibiotics. $52.6 \%$ of the neonates were term and $47.3 \%$ were preterm. Amikacin (93.4\%) followed by Cefotaxime (68.4\%) and Ampicillin (31.6\%) were the most common antimicrobials used. $52.6 \%$ did not respond to first line of antibiotics whereas only $47.4 \%$ responded to first line drugs. Conclusion: Majority of the infants (75\%) admitted in the NICU received antibiotics during their course of treatment. Greater number of Preterm neonates received antibiotics in comparison to Term neonates. There is decrease in susceptibility of organisms to first line antibiotics leading to exposure of neonates to multiple $(>2)$ antibiotics.

Key Word: antimicrobial pattern.

*Address for Correspondence:

Dr Abhishek Patel, Assistant Professor, Department of Pediatrics, Navodaya Medical College, Raichur, Karnataka, INDIA.

Email: ajayj1987@gmail.com

Received Date: 17/12/2019 Revised Date: 13/01/2020 Accepted Date: 06/02/2020

DOI: https://doi.org/10.26611/10141521

This work is licensed under a Creative Commons Attribution-NonCommercial 4.0 International License.

\begin{tabular}{|l|l|}
\hline \multicolumn{2}{|c|}{ Access this article online } \\
\hline Quick Response Code: & Website: \\
www.medpulse.in \\
\hline
\end{tabular}

\section{INTRODUCTION}

Antimicrobials are the most common medications used in NICU. Most often they are prescribed as a part of treatment for sepsis. They are also administered empirically in neonates during their hospital stay even without sepsis ${ }^{1}$. The western countries have moved from empirical approach to evidence based approach but the literature review in developing countries shows the prevalence of irrational prescription of antibiotics because of empirical approach which is driven by the economic constraints of the patients ${ }^{2}$. There is increased risk of invasive candidiasis and death with use of broad spectrum antibiotics and there is also a risk of necrotizing enterocolitis, late onset sepsis and death with prolonged duration of antibiotics ${ }^{3}$. There is lack of safety, dosing and efficacy of drugs studies in neonates due to ethical concerns and difficulties in recruitment ${ }^{4}$. Further the pre-terms admitted in NICU can be associated with renal and liver dysfunction which excludes them from the trails. These factors have led the clinicians for off-label use of medications ${ }^{5}$. The prolonged and inadvertent use of antibiotics in neonates and especially in pre-term neonates requiring prolonged hospitalization can lead to antibiotic resistance, fungal infections, prolonging hospital stay and also increase in mortality. Further delay in the start on antibiotics in sepsis can lead to increase in mortality of the neonates ${ }^{6}$. There are no standard guidelines for prescribing antibiotics in NICU's in India. The choice of antibiotics is driven by the prevailing neonatal problems in the region. There are is also no much data available on the pattern of antibiotic 
usage in neonates. Hence the study was conducted to assess the pattern of antibiotic usage in our neonatal intensive care unit.

\section{METHODOLOGY}

This is a retrospective observational study conducted in a tertiary care hospital in south India.

The data was collected retrospectively and all the neonates treated with antibiotics for three months were included in the study. The pattern of antimicrobial usage pattern in NICU was studied retrospectively in a tertiary care teaching hospital.

Those neonates without antibiotics were excluded from the study.

RESULTS: A total of 102 neonates were included in the study

Table 1: showing proportion of neonates receiving antibiotics

\begin{tabular}{cc}
\hline Parameter & Total \\
\hline Admissions to NICU & 102 \\
Neonates received antibiotics & $76(74.5 \%)$ \\
\hline
\end{tabular}

From the above table, of the 102 admissions, 76 (74.5\%) neonates required antibiotic

Table 2: showing gestational age of the neonates receiving

\begin{tabular}{cc}
\multicolumn{2}{c}{ antibiotics } \\
\hline Gestation & Number \\
\hline Term & $40(52.6 \%)$ \\
Late preterm & $22(28.9 \%)$ \\
Early preterm & $14(18.4 \%)$ \\
\hline Total & 76 \\
\hline
\end{tabular}

From the above table $52.6 \%$ of neonates were term and $47.3 \%$ were preterm.

Table 3: showing gender distribution among neonates receiving

\begin{tabular}{cc}
\multicolumn{2}{c}{ antibiotics } \\
\hline Gender & Total \\
\hline Male & $40(52.6 \%)$ \\
Female & $36(47.4 \%)$ \\
\hline
\end{tabular}

Table 4: showing antibiotic profile

\begin{tabular}{ccc}
\hline Generic name with dosage & Dosage & No. of subjects (\%) \\
\hline Cefotaxime & $50 \mathrm{mg} / \mathrm{Kg}$ IV BID & $52(68.4)$ \\
Amikacin & $7.5 \mathrm{mg} / \mathrm{Kg}$ IV BID & $71(93.4)$ \\
Ampicillin & $50 \mathrm{mg} / \mathrm{kg} \mathrm{IV} \mathrm{BID}$ & $24(31.6)$ \\
Ceftriaxone & $50 \mathrm{mg} / \mathrm{kg}$ IV BID & $9(11.8)$ \\
Amoxicillin +Clavulanic Acid & $50 \mathrm{mg} / \mathrm{kg}$ IV BID & $28(36.8)$ \\
Piperacillin + Tazobactum & $100 \mathrm{mg}$ IV BID & $19(25)$ \\
Metronidazole & $7.5 \mathrm{mg} / \mathrm{kg}$ IV TID & $6(7.9)$ \\
Meropenem & $20 \mathrm{mg} / \mathrm{kg}$ IV BID & $7(9.2)$ \\
Vancomycin & $40 \mathrm{mg} / \mathrm{kg}$ IV QID & $6(7.9)$ \\
Colistin & 50,000 IU/kg TID & $5(6.6)$ \\
\hline
\end{tabular}

Among the empirically used antibiotics most common was Amikacin 71(93.4\%) followed by Cefotaxime 52(68.4\%) and Ampicillin 24(31.6\%).

\begin{tabular}{cc}
\multicolumn{3}{c}{ Table 5: showing change in antibiotics } \\
\hline Change in antibiotics & Number \\
\hline Yes & $\mathbf{4 0 ( 5 2 . 6 \% )}$ \\
No & $\mathbf{3 6 ( 4 7 . 4 \% )}$ \\
\hline Total & 76
\end{tabular}

From the above table $40(52.6 \%)$ neonates required change in antibiotics.

\begin{tabular}{cc} 
Table 6: showing reasons for change in antibiotics \\
\cline { 2 - 3 } Reason for change & Number \\
\hline No clinical response & $\mathbf{1 1 ( 2 7 . 5 \% )}$ \\
Culture report & $\mathbf{1 7 ( 4 2 . 5 \% )}$ \\
Specific indication & $\mathbf{1 2 ( 3 0 \% )}$ \\
\hline Total & 40
\end{tabular}

From the above table the most common reason for change in antibiotics was Culture and sensitivity report.

\section{DISCUSSION}

In our study, 76(74.5\%) neonates out of 102 admissions received antibiotics. This finding comparable to the observations of study by Sonali Suryavanshi et al. in a tertiary care centre Pune, India who observed that antibiotics was administered in $70 \%$ of the neonates ${ }^{7}$. In another study by Camila Hauge et al. a study conducted at Ujjain, India showed usage of antibiotics in $71-89 \%$ of neonates ${ }^{8}$.In another study by Megha Sharma et al. antibiotics was used in $79-82 \%$ of neonates ${ }^{9}$.

Among the neonates included in the study, term neonates were $40(52.6 \%)$ and $36(47.3 \%)$ were preterm infants. This finding is comparable to the observations of study by Sonali Suryavanshi et al. in which 246 (46.6\%) were term and $282(53.4 \%)$ were preterm ${ }^{7}$. Among the empirically used antibiotics most common was Amikacin 71(93.4\%) followed by Cefotaxime 52(68.4\%) and Ampicillin $24(31.6 \%)$. In a study by Sonali Suryavanshi et al. the majority of neonates $(55.9 \%)$ received between 1-2 antibiotics, Amikacin, Cefotaxime and Levofloxacin were the drugs most often prescribed ${ }^{7}$. The most commonly prescribed antibiotic groups in a study by Megha Sharma et al. aminoglycosides, cephalosporins and fluroquinolones ${ }^{9}$. In a review of antibiotic usage in NICU by found the common empirical antibiotics used were cefotaxime, ampicillin and aminoglycosides similar to our study ${ }^{10}$. In our study $36(47.4 \%)$ neonates responded to first line of antibiotics, while $40(52.6 \%)$ neonates required change in antibiotics. The most common reason for change in antibiotics was Culture and sensitivity pattern followed by specific indication like NEC, Arthritis, Meningitis. In $11(27.5 \%)$ antibiotic was changed due to lack of clinical response. In studies from UK by Muller-Pebody $\mathrm{B}^{11}$ and Italy by Chryssoula Tzialla ${ }^{1}$ it was noted that more than $90 \%$ of the organisms were sensitive to first line of antibiotics. Where as in our study we noted that only $47.4 \%$ responded to first line antibiotics, which was similar 
to another Indian study by Sonali Suryavanshi et al.. This suggests a rising trend in drug resistance among organisms isolated from NICU.

\section{CONCLUSION}

Majority of the infants (75\%) admitted in the NICU received antibiotics during their course of treatment. Greater number of Preterm neonates received antibiotics in comparison to Term neonates. There is decrease in susceptibility of organisms to first line antibiotics leading to exposure of neonates to multiple $(>2)$ antibiotics.

\section{REFERENCES}

1. Chryssoula Tzialla, Alessandro Borghesi, Gregorio Serra, et al.. Antimicrobial therapy in neonatal intensive care unit Italian Journal of $P$ ediatrics. 2015;41:27

2. Le Grand A, Hogerzeil HV, Haaijer-Ruskamp FM. Intervention research in rational use of drugs: A review. Health Policy Plan 1999;14(2):89-102

3. Tripathi N, Cotten CM, Smith PB. Antibiotic use and misuse in the neonatal intensive care unit. Clin Perinatol. 2012;39(1):61-8. doi: 10.1016/j.clp.2011.12.003

4. Bavdekar S, Gogtay N. Unlicensed and off-label drug use in children. J Postgrad Med. 2005; 51:249-252. [PubMed: 16388164]
5. Pandolfini C, Bonati M. A literature review on off-label drug use in children. Eur J Pediatr. 2005; 164:552-558. [PubMed: 15912383]

6. Cotten CM, Taylor S, Stoll B, et al.. Prolonged duration of initial empirical antibiotic treatment is associated with increased rates of necrotizing enterocolitis and death for extremely-low-birth-weight infants. Pediatrics. 2009; 123(1):58-66. [PubMed: 19117861]

7. Suryawanshi S, Pandit V, Suryawanshi P, Panditrao A. Antibiotic Prescribing Pattern in a Tertiary Level Neonatal Intensive Care Unit. J Clin Diagn Res. 2015;9(11):FC21FC24. doi:10.7860/JCDR/2015/14764.6807

8. Hauge C, Stålsby Lundborg C, Mandaliya J, Marrone G, Sharma M. Up to $89 \%$ of neonates received antibiotics in cross-sectional Indian study including those with no infections and unclear diagnoses. Acta Paediatr. 2017;106(10):1674-1683. doi:10.1111/apa.13935

9. Sharma M, Eriksson B, Marrone G, Dhaneria S, Lundborg CS. Antibiotic prescribing in two private sector hospitals; one teaching and one non-teaching: a cross-sectional study in Ujjain, India. BMC Infect Dis. 2012;12:155. Published 2012 Jul 12. doi:10.1186/1471-2334-12-155.

10. Tzialla C, Borghesi A, Serra G, Stronati M, Corsello G. Antimicrobial therapy in neonatal intensive care unit. Ital $J$ Pediatr. 2015;41:27. Published 2015 Apr 1. doi:10.1186/s13052-015-0117-7

11. Muller-Pebody B, Johnson AP, Heath PT, et al. . Empirical treatment of neonatal sepsis: are the current guidelines adequate? Arch Dis Child Fetal Neonatal Ed. 2011; 96(1):F4-F8. [PubMed: 20584804]

\section{Source of Support: None Declared Conflict of Interest: None Declared}

Policy for Articles with Open Access:

Authors who publish with MedPulse International Journal of Pediatrics (Print ISSN: 2579-0897) (Online ISSN: 2636-4662) agree to the following terms: Authors retain copyright and grant the journal right of first publication with the work simultaneously licensed under a Creative Commons Attribution License that allows others to share the work with an acknowledgement of the work's authorship and initial publication in this journal.

Authors are permitted and encouraged to post links to their work online (e.g., in institutional repositories or on their website) prior to and during the submission process, as it can lead to productive exchanges, as well as earlier and greater citation of published work. 